\title{
Looking Around to Look Forward
}

Monitoring should be an obsession in the management of mechanically ventilated patients. The control of vital signs and respiratory parameters must guide the ICU professional in responding with precision to the patient's needs. The setting of mechanical ventilation parameters is a very important issue in the management of a critically ill patient, and the setting of the ideal PEEP level is a particular chapter that has been largely investigated and is still challenging. The strategy used to set it properly can vary from one center to another, always depending on the ICU's economic condition and the professionals' skills. ${ }^{1-3}$

In this issue of RESPIRATORY CARE, the study by Sindi et $\mathrm{al}^{4}$ contributes to the knowledge of respiratory mechanics and, furthermore, carries a very important message to the ICU health care professional. The authors studied surgical patients who underwent laparoscopy, exploring the correlation between abdominal pressure $\left(\mathrm{P}_{\mathrm{abd}}\right)$ and esophageal pressure $\left(\mathrm{P}_{\mathrm{es}}\right)$. As $\mathrm{P}_{\mathrm{es}}$ is an alternative for estimation of pleural pressure $\left(\mathrm{P}_{\mathrm{pl}}\right)$, the correlation of $\mathrm{P}_{\mathrm{abd}}$ with $\mathrm{P}_{\mathrm{es}}$ should be useful to obtain information on $\mathrm{P}_{\mathrm{pl}}$ bypassing the measurement of $\mathrm{P}_{\mathrm{es}}$ in these patients. The measurement of $\mathrm{P}_{\mathrm{es}}$ and $\mathrm{P}_{\mathrm{pl}}$ to set the PEEP has already been proposed. . $^{5-7}$ Unlike other trials, the protocol proposed by Sindi et al measured $\mathrm{P}_{\mathrm{abd}}$ in patients with neuromuscular blockade, which eliminates the effect of the diaphragmatic tone and allows a more reliable estimation of $\mathrm{P}_{\mathrm{pl}}$. Their results show us that $\mathrm{P}_{\mathrm{abd}}$ may provide useful complementary information in the management of mechanically ventilated patients.

Beyond the main goal, there is a hidden objective, which is stated in the last paragraph of the introduction, that I want to address: "stimulate researchers to look at the use of bedside abdominal pressure measurements." Why is this important? The study by Sindi et $\mathrm{al}^{4}$ encourages us to look around and to give value to all bedside information available when the patient is mechanically ventilated. Looking forward, Sindi et al show us that, at least in some clinical settings, the assessment of $\mathrm{P}_{\mathrm{abd}}$ by bladder pressure measurement could be helpful to estimate $\mathrm{P}_{\mathrm{pl}}$ to set

The author has disclosed no conflicts of interest.

Correspondence: Camilo Corbellini MSc PT, Via Napoleone Leumann 24, CAP 10098 Rivoli (TO), Italy. E-mail: camilocorbellini@hotmail. com.

DOI: $10.4187 /$ respcare.03239 the PEEP level safely, avoiding the potential problems and risks of esophageal probe insertion and proposing a solution to the problem of a lack of familiarity with this technique. This is a dilemma of $\mathrm{P}_{\mathrm{es}}$ and $\mathrm{P}_{\mathrm{abd}}$ monitoring. The interpretation of information regarding the measurement of $\mathrm{P}_{\mathrm{es}}$ and $\mathrm{P}_{\mathrm{abd}}$ must be analyzed carefully. If we ignore $\mathrm{P}_{\mathrm{abd}}$, for example, we could also ignore its effect on $\mathrm{P}_{\mathrm{pl}}$ and its implications in the management of mechanically ventilated patients. ${ }^{7}$ Regarding the field of monitoring ICU patients, the authors make a very important point: different instruments and parameters may be considered for deciding the setting of mechanical ventilation. Any parameter that is just in front of us must not be denied or underestimated. Relevant information may be easy to find if we take a careful look around.

The authors also state that their results were too limited to have general value. ${ }^{4}$ This is obviously true, considering the characteristics of their sample. However, in the end, the correlation found opens a new track to future investigations on the adjustment and monitoring of the ideal PEEP level. This is also looking forward. Sindi et al ${ }^{4}$ understood that, as explained by Sarge and Talmor, ${ }^{8}$ the airway pressure itself will fail to separate severe perturbations in the elastance of the respiratory system when we titrate the PEEP level, and that is why their results are important: the health care professional must have alternative or complementary measurements to achieve this important goal.

Camilo Corbellini MSc PT $\mathrm{PhD}$ student at Università degli Studi di Milano Milan, Italy and Department of Pulmonary Rehabilitation Casa di Cura Villa Serena Turin, Italy

\section{REFERENCES}

1. Marini J. How to recruit the injured lung. Minerva Anestesiol 2003; 69(4):193-200.

2. Chiumello D, Cressoni M, Carlesso E, Caspani ML, Marino A, Gallazzi E, et al. Bedside selection of positive end-expiratory pressure in mild, moderate, and severe acute respiratory distress syndrome. Crit Care Med 2014;42(2):252-264.

3. Keenan JC, Dries DJ. PEEP titration: new horizons. Respir Care 2013;58(9):1552-1554. 


\section{LOOKING AROUND to LOOK Forward}

4. Sindi A, Piraino T, Alhazzani W, Tunks M, Faden M, Ma J, et al. The correlation between esophageal and abdominal pressures in mechanically ventilated patients undergoing laparoscopic surgery. Respir Care 2014;59(4):491-496.

5. Talmor D, Sarge T, Malhotra A, O’Donnell CR, Ritz R, Lisbon A, et al. Mechanical ventilation guided by esophageal pressure in acute lung injury. N Engl J Med 2008;359(20):2095-2104.

6. Gulati G, Novero A, Loring SH, Talmor D. Pleural pressure and optimal positive end-expiratory pressure based on esophageal pres- sure versus chest wall elastance: incompatible results. Crit Care Med 2013;41(8):1951-1957.

7. Loring SH, O'Donnell CR, Behazin N, Malhotra A, Sarge T, Ritz R, et al. Esophageal pressures in acute lung injury: do they represent artifact or useful information about transpulmonary pressure, chest wall mechanics, and lung stress? J Appl Physiol 2010;108(3):515-522.

8. Sarge T, Talmor D. Transpulmonary pressure: its role in preventing ventilator-induced lung injury. Minerva Anestesiol 2008;74(6):335339 . 